Gut, 1983, 24, 120-126

\title{
Small intestinal nodular lymphoid hyperplasia in patients with giardiasis and normal serum immunoglobulins
}

\author{
H WARD, K N JALAN, T K MAITRA, S K AGARWAL, AND D MAHALANABIS \\ From the Calcutta Medical Research Institute and Kothari Centre of Gastroenterology, Calcutta, India
}

SUMmaRY Nodular lymphoid hyperplasia of the upper small intestine was demonstrated in 25 patients with giardiasis. All had normal serum immunoglobulin levels and seven patients initially presented with clinical findings suggestive of an abdominal lymphoma. In only two, however, was the diagnosis of primary jejunal lymphoma confirmed. It is possible that an aetiological relationship exists between recurrent parasitic infestation and nodular lymphoid hyperplasia of the upper small intestine.

The association between nodular lymphoid hyperplasia (NLH) of the upper small intestine and parasitic infestation, particularly with giardiasis, was initially described in patients with humoral immunodeficiency disorders.' Subsequently, lymphoid nodules have been described in the terminal ileum ${ }^{2}$ and colon ${ }^{3}$ of immunologically normal children, but nodular lymphoid hyperplasia of the upper small intestine has only rarely been observed in the absence of humoral immunodeficiency. ${ }^{4}$

In this report we describe a series of 25 patients with giardiasis who had normal serum immunoglobulins and nodular lymphoid hyperplasia of the upper small intestine. It was suspected, on clinical grounds, that seven of these patients had an intestinal lymphoma; in only two, however, was this diagnosis histologically confirmed.

\section{Methods}

\section{PATIENTS}

Twenty-five patients (six females and 19 males, age range 12 to 42 years, mean $26 \cdot 36$ years) were studied over a three year period. Patients were divided into two groups according to their clinical presentation.

\section{GROUP 1 (cases 1-18)}

The duration of symptoms in patients in group 1 ranged from three months to 10 years. Patients presented with a history of recurrent diarrhoea or

Reccived for publication $7 \mathrm{Mas}$ 19x2 alternating diarrhoea and constipation, anorexia, weight loss, and generalised weakness. Physical examination revealed no significant abnormalities apart from those of mild nutritional deficiencies such as pallor and glossitis. Most of the patients gave a history of recurrent giardiasis with symptom-free intervals after antigiardial therapy.

\section{GROUP 2 (cases 19-25)}

All patients in this group were suspected of having an intestinal lymphoma. The duration of symptoms ranged from six months to 13 years. They gave a history of symptoms similar to those in group 1, but, in addition, complained of marked weight loss, fever, and abdominal pain. On examination they were emaciated, and had pallor and glossitis. Two patients had clubbing and pedal oedema. All patients had palpable abdominal masses suggestive of lymphadenopathy: two had hepatomegaly and one had splenomegaly. Six patients were subjected to exploratory laparotomy. At operation five were found to have no gross abnormality apart from enlarged mesenteric lymph nodes. Small intestinal biopsies were taken. Patient no. 24 in this group presented with a 10 year history of recurrent giardiasis, and a barium meal study done four years before admission was suggestive of nodular lymphoid hyperplasia. An irregular hard mass was palpable in the upper abdomen; at surgery this proved to be a lymphoblastic lymphoma arising from the upper jejunum. The rest of the intestine was studded with multiple nodules, some of which showed malignant change. 
Patient no. 25 was admitted with a seven year history of recurrent giardiasis. Barium meal examination showed a markedly disordered small intestinal pattern with numerous rounded filling defects suggestive of nodular lymphoid hyperplasia. Peroral jejunal biopsy revealed a diffuse histiocytic lymphoma. This patient refused surgery and was subsequently lost to follow up.

\section{INVESTIGATIONS}

In addition to routine haematological and biochemical investigations the stools were examined for parasites on three separate days. In 22 patients a sample of the jejunal aspirate was examined for parasites and a second sample was cultured quantitatively for aerobic and anaerobic bacteria. ${ }^{5}$ Small intestinal absorption was assessed by urinary D-xylose excretion after a $5 \mathrm{~g}$ oral dose, ${ }^{6}$ the Schilling test, ${ }^{7}$ and 72 hours faecal fat excretion on a $75 \mathrm{~g}$ fat diet. ${ }^{8}$

Serum (in all patients) and jejunal fluid (in 15 patients) immunoglobulins were measured by single radial immunodiffusion, ${ }^{9}$ using WHO reference sera as standards and antisera obtained from Behringwerke. Upper gastrointestinal barium meal studies were performed in all patients. Peroral jejunal biopsy was obtained using a Crosby capsule. In patients in group 2, sera were tested for the presence of free alpha heavy chains by an immunoselection plate technique, ${ }^{11}$ using antihuman kappa and antihuman lambda antisera (Behringwerke) in the gel and an antihuman alpha chain specific antiserum (Behringwerke) in the trough. Operative specimens of lymph node and jejunal biopsies were subjected to histopathological examination. In selected patients, paraffin-embedded sections of jejunal biopsies were stained by an unlabelled antibody enzyme immunoperoxidase technique ${ }^{11}$ (using a peroxidase-antiperoxidase soluble immune complex prepared in this laboratory and antisera obtained from Behringwerke) for $\operatorname{IgA}, \operatorname{IgG}$, and $\operatorname{IgM}$ containing cells.

Nineteen patients were treated with a course of metronidazole $(1200 \mathrm{mg} /$ day) for seven to 10 days and stool examination and absorption studies (which were abnormal before treatment) were repeated. All 19 patients who had repeat studies had a minimum of two jejunal biopsies repeated two to three weeks after a course of metronidazole. The patients in whom partial atrophy of the villi and malabsorption persisted after antigiardial treatment were presumed to have an underlying primary tropical malabsorption syndrome and were treated with a long-term course of tetracycline and folic acid and jejunal biopsies repeated three weeks, and. in some. six weeks after starting treatment. Barium meal studies were not repeated after treatment as we did not feel that it would be justified to repeat this investigation within such a short time. Moreover, not all patients had radiographic changes of nodular lymphoid hyperplasia.

\section{Results}

Results of relevant investigations are summarised in the Table.

All patients in both groups had Giardia lamblia in the stool, and/or jejunal aspirate. Six of 22 patients had a significant bacterial overgrowth with counts $>10^{5} / \mathrm{ml}$ in the upper jejunum. Twenty patients had malabsorption of one or more test substances. Serum immunoglobulin levels in all patients were within control limits for this population. Jejunal fluid immunoglobulin levels in 15 patients did not differ significantly from controls. Free alpha heavy chains were not detectable in any of the sera tested. Barium series revealed a disordered small intestinal pattern with coarsening and widening of the jejunal loops in 21 patients. In 14 patients, small regular filling defects could be seen in the jejunum (Fig. 1). Jejunal biopsies were independently assessed by two reviewers. Nodular lymphoid hyperplasia was defined as the presence of one or more large aggregates of lymphoid cells in the lamina propria or submucosa. Serial sections through these aggregates in most biopsies revealed a follicular structure with a hyperplastic germinal centre and a surrounding rim of small lymphocytes (Fig. 2). In some sections these follicles were situated deep in the lamina propria or submucosa while in others they protruded into the lumen causing effacement of overlying villi. An increase in the cellularity of the lamina propria and an increase in intraepithelial lymphocytes was present in all biopsies. A consistent finding was the presence of normal numbers of plasma cells in all biopsies. Staining by the unlabelled enzyme antibody technique revealed an abundance of $\mathrm{Ig}$ bearing cells in the lamina propria, most of which contained either $\operatorname{IgM}$ or $\operatorname{IgA}$ in almost equal proportions; there were very few IgG bearing cells. The majority of cells in the lymphoid follicles were found to be non-Ig bearing cells. A few IgA (Fig. 3) and IgM bearing cells, however, were seen in the follicles. Histological examination of enlarged mesenteric lymph nodes in cases 19-25 revealed reactive follicular hyperplasia.

There was no definite correlation between clinical symptoms, absorption parameters. and histological changes in patients in both groups.

RESPONSE TO TREATMENT

Repeat stool examination was negative in all 


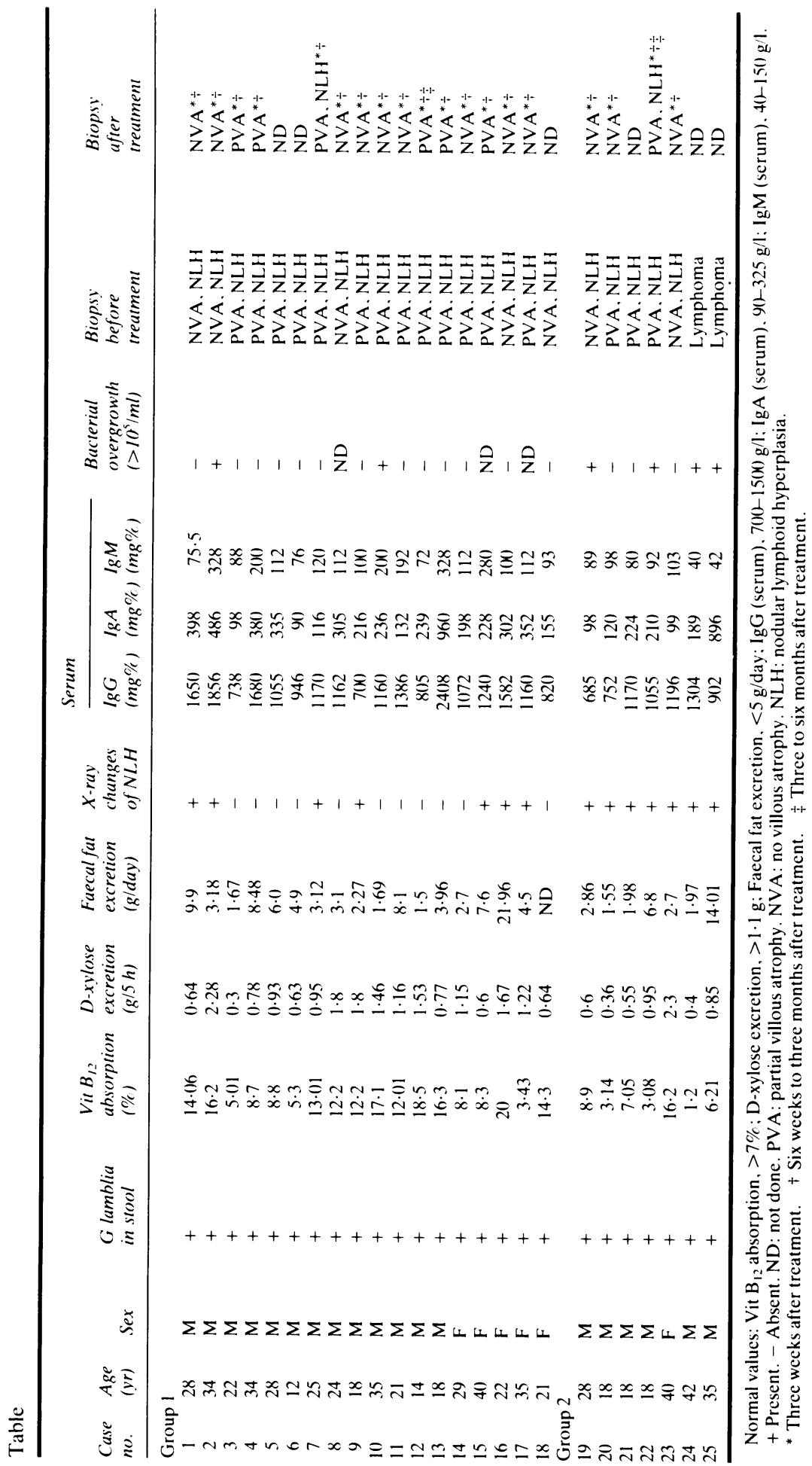




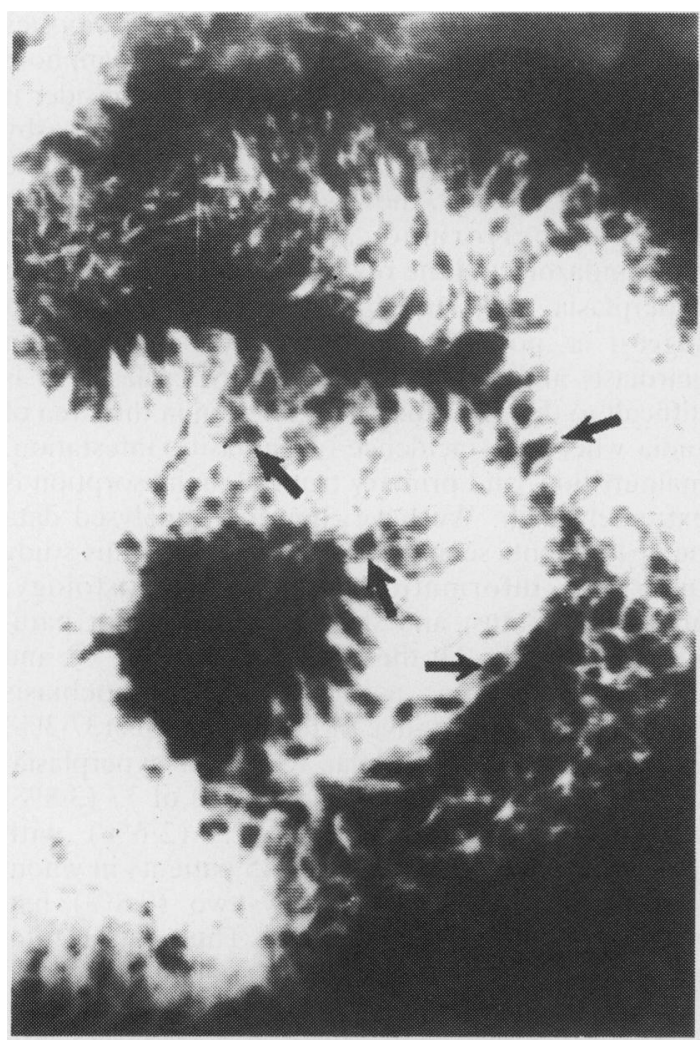

Fig. 1 Barium meal study of patient with nodular lymphoid hyperplasia: small rounded filling defects can be seen in jejunal loops.

patients who were treated with metronidazole. Twelve of the 19 patients improved symptomatically after treatment. In seven patients, bowel symptoms improved but anorexia and weight loss persisted. These seven patients were found to have persistent partial atrophy of the villi and malabsorption, and were presumed to have an underlying primary tropical malabsorption syndrome; they were subsequently treated with tetracycline and folic acid. In 17 of the 19 patients changes of nodular lymphoid hyperplasia could not be detected in two or more jejunal biopsies repeated after treatment.

\section{Discussion}

Certain features distinguish this group of patients from those with the classical syndrome of nodular lymphoid hyperplasia, which has been described in patients with panhypogammaglobulinaemia or selective IgA deficiency. ${ }^{1}$ In this syndrome, jejunal

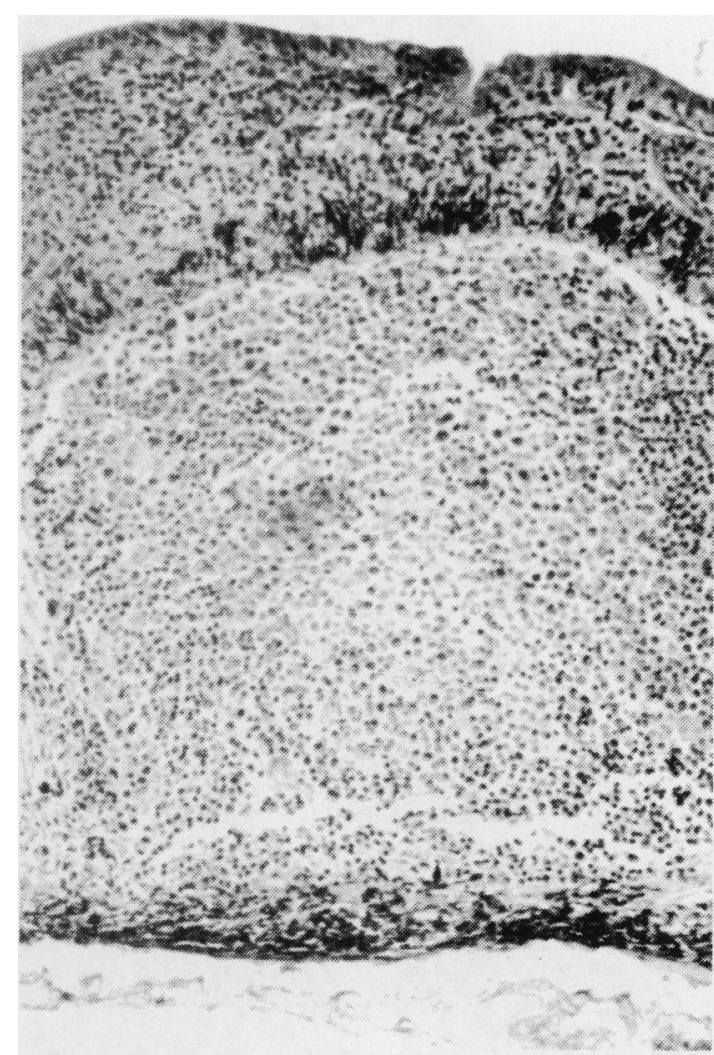

Fig. 2 Section from jejunal biopsy showing a hyperplastic lymphoid follicle. $H$ and $E, \times 125$ (original magnification).

villus architecture is generally normal, bacterial overgrowth in the jejunum is common, and plasma cells are absent or deficient in the lamina propria. ${ }^{12}$ Immunofluorescence studies of the jejunal mucosa reveal an almost total absence of Ig bearing cells in the lamina propria. ${ }^{13}$ Nodular lymphoid hyperplasia in these patients is believed to represent either an accumulation of plasma cell precursors because of a maturational defect in the B lymphocyte series or a florid local immune response to unknown antigens. ${ }^{414}$ Giardiasis has generally been assumed to be secondary to the immunodeficiency state. Although eradication of the parasite results in clinical improvement, a change in the size or number of the nodules has not been observed. ${ }^{12}$ None of the patients in our study had a severe humoral immunodeficiency disorder. The majority had partial atrophy of the villi and malabsorption. Plasma cells were present in normal numbers and immunoperoxidase staining of the jejunal mucosa 


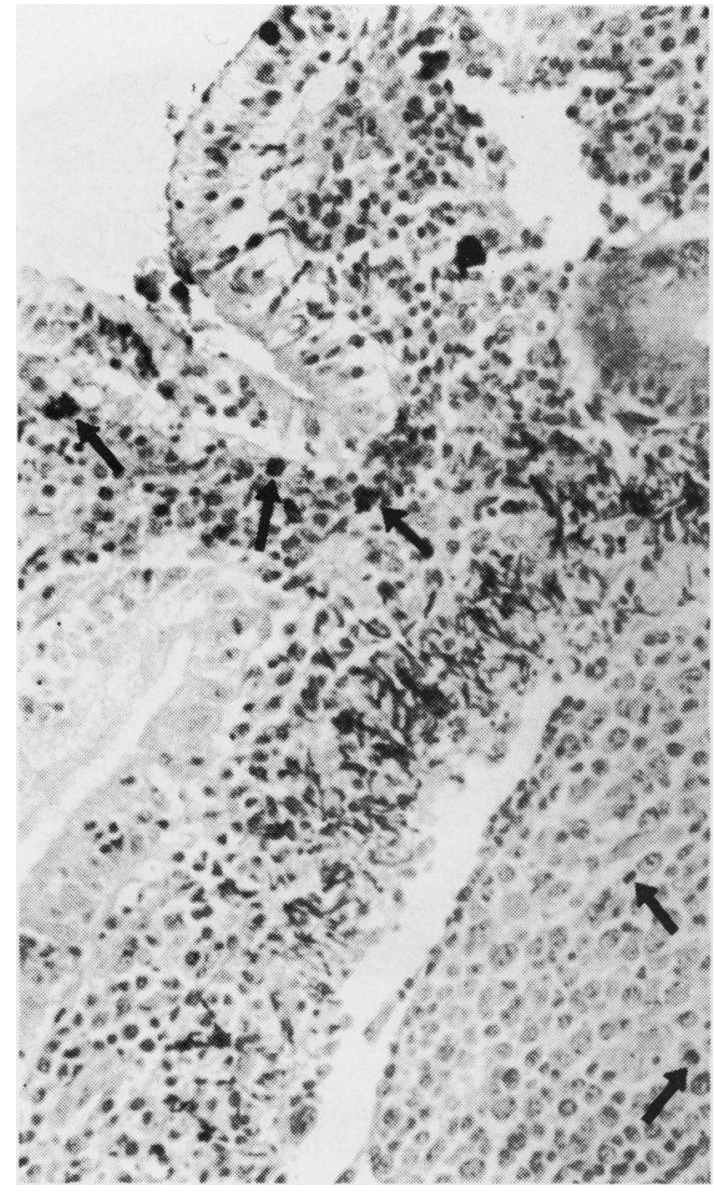

Fig. 3 Section from jejunal biopsy showing IgA bearing cells in a lrmphoid follicle and adjoining lamina propria. Immunoperoxidase. $\times 25()$ (original magnification).

revealed an abundance of $\operatorname{IgA}$ and $\operatorname{IgM}$ bearing cells in the lamina propria. The incidence of bacterial overgrowth in the jejunum was similar to that of patients with giardiasis without nodular lymphoid hyperplasia. but was higher than that of patients with other causes of diarrhoea (see below). Architectural changes in the villi improved in only 12 of 19 patients but nodular lymphoid hyperplasia apparently disappeared in all but two. Persistence of anorexia. weight loss. and partial atrophy of the villi even after metronidazole therapy in seven of the 19 patients raises the possibility of an underlying primary tropical enteropathy in association with giardiasis. This diagnosis was supported by their subsequent improvement on a regime of tetracycline and folic acid.

It is well-known that nodular lymphoid hyper- plasia may be a patchy lesion and multiple biopsies are often necessary to confirm reversal of lymphoid hyperplasia after treatment. ${ }^{+}$We did not consider it ethical to perform multiple biopsies with a Crosby capsule nor would it have been acceptable to the patients. They did, however, have at least two biopsies performed after treatment with metronidazole and the reversal of nodular lymphoid hyperplasia after antigiardial therapy appears to suggest a possible causal relationship between giardiasis and nodular lymphoid hyperplasia. It is difficult to define a control population in this area of India where the incidence of paraasitic infestation. malnutrition. and primary tropical malabsorption is extremely high. We have, however, analysed data on 464 patients seen during the period of this study in whom information on jejunal histology, absorption status, and stool examination for parasites is available. Of these 464 patients, 67. 77, and 75 had giardiasis, ascariasis, and amoebiasis respectively. Twenty-five of the 67 patients $(37 \cdot 3 \%)$ with giardiasis had nodular lymphoid hyperplasia, whereas this was found in only three of $77(3.8 \%)$ with ascariasis and two of $75(2.6 \%)$ with amoebiasis. Of the remaining 245 patients in whom there were no parasites, only two $(0.8 \%)$ had nodular lymphoid hyperplasia. Thus, of a total number of 32 patients with nodular lymphoid hyperplasia seen during this period. $25(78 \cdot 1 \%)$ had giardiasis. These data further corroborate our speculation regarding the relationship of giardiasis to nodular lymphoid hyperplasia. It is, however, possible that other organisms, as yet unidentified, may be responsible for the lymphoid hyperplasia.

Wright and Tomkins ${ }^{15}$ have demonstrated that patients with giardiasis and malabsorption have increased numbers of intraepithelial lymphocytes in the small intestine which decrease after antigiardial therapy. They have drawn an analogy to a similar phenomenon in coeliac disease and suggest that a hypersensitivity response to a giardial antigen (similar to that produced by gluten) may be responsible for the mucosal damage and malabsorption. It is possible that the lymphoid hyperplasia in our patients represents a similar hypersensitivity response to chronic antigenic stimulation by Giardia lamblia. It is of interest that lymphoid follicles have also been frequently observed in biopsies from children with giardiasis. ${ }^{16}$ Owen et $a l^{17}$ in studies of Giardia muris infested mice. have also observed large numbers of small lymphocytes in the mucus of the intestinal lumen, some of which were adherent to the Giardia trophozoites. They suggested that this represents an immune mediated migration of lymphocytes which may help eliminate the parasite. 
Although all our patients had normal serum immunoglobulins we have not excluded the possibility of a functional antibody deficiency. particularly as some of them were severely malnourished. Although this is unlikely, it is a factor that needs to be further evaluated.

Our finding of a relative increase in IgM bearing cells in the lamina propria confirms the observations of Ridley and Ridley: ${ }^{18}$ patients with systemic humoral immunodeficiency have also been shown by Webster et $\mathrm{al}^{14}$ to produce IgM locally in the gut.

Two patients in group 2 were found to have a primary upper gastrointestinal lymphoma. Both patients have a long history of recurrent giardiasis and one patient had radiological features suggestive of nodular lymphoid hyperplasia four years before the diagnosis of lymphoma. A similar case of nodular lymphoid hyperplasia and lymphoma in a normo-gammaglobulinaemic patient has been described by Kahn et al. ${ }^{19}$ Shaw and Hennigar 211 have described the occurrence of nodular lymphoid hyperplasia in association with lymphoma outside the gastrointestinal tract. The association between nodular lymphoid hyperplasia and jejunal lymphoma has also been described in a patient with adult onset hypogammaglobulinaemia. ${ }^{2}$

The clinical picture in patients in group 2 is similar in many respects to that of patients with alpha heavy chain disease. It is postulated that an unknown chronic antigenic stimulus underlies this disease. ${ }^{22}$ Giardiasis is commonly found in these patients, although no consistent association with any pathogen has been noted. It is interesting that in some cases of alpha heavy chain disease the initial biopsy of the jejunum and/or mesenteric lymph nodes shows a non-specific hyperplastic lymphoid infiltration. ${ }^{23}$ Subsequently a plasma cell infiltrate becomes evident. Similar lymphoproliferation is associated with dilantin therapy ${ }^{24}$ and coeliac disease $^{25}$ where there is a spectrum ranging from a benign non-specific reaction to overt malignancy. Whether a genetic predisposition or an unknown stimulus could trigger the malignant potential of these disorders remains speculative.

We are grateful to C R Dutta, B Biswas, N C Bose, and A Mohimen for technical assistance; Dr Shiv Pillai for help with the peroxidase conjugates and immunoselection plate technique, and Mr E G P Nair for secretarial assistance.

\section{References}

1 Hermans PE, Huizenga KA, Hoffman HN, II et al. Dysgammaglobulinaemia associated with nodular lymphoid hyperplasia of the small intestine. Am J Med 1966; 40: 78-89.

2 Fieber SS, Schacfer HJ. Lymphoid hyperplasia of the terminal ileum - a clinical entity. Gastroenterology 1966: 50: 83-98.

3 Capitano MA. Kirkpatrick JA. Lymphoid hyperplasia of the colon in children. Radiology 1970; 94: 323-7.

4 Webster ADB. The gut and immunodeficiency disorders. In: Clinics in gastroenterology. Philadelphia: Saunders. 1976, vol 5, no. 2: 333.

5 Gorbach SL, Jacobs B. Mitra R et al. Bacterial contamination of the upper small bowel in tropical sprue. Lancet 1969: 1: 74-7.

6 Santini R. Jr. Sheehy TW. Martinez-de-Jesus J. The xylose tolerance test with a $5 \mathrm{~g}$ dose. (jastroenterology 1961: 40: 772-4.

7 Schilling RF. Intrinsic factor studies. II. The effect of gastric juice on the urinary excretion of radio-activity after the oral administration of radioactive vitmin $\mathrm{B}_{12}$. $J$ Lab Clin Med 1953; 42: 86()-6.

8 Henry RJ. In: Clinical chemistry. New York: Harper and Row, 1966.

9 Fahey JL. McKelvey EM. Quantitative determination of serum immunoglobulins in antibody agar plates. $J$ Immunol 1965; 94: 84-90).

1) Doe WF. Henry K. Holbo JR et al. Five cases of alpha chain disease. Gut 1972; 13: 947-53.

11 Sternberger LA. Hardy PH. Cuculis TJ et al. The unlabelled antibody enzyme method of immunohistochemistry. Preparation and properties of soluble antigen antibody (horse radish peroxidase-antihorse radish peroxidase) and its use in identification of spirochaetes. J Histochem (ytochem 1970; 18: 315-33.

12 Ochs HD, Ament ME. Gastrointestinal tract and immunodeficiency. In: Ferguson A. MacSween RN. eds. Immunological aspects of the liver and gastrointestinal tract. Lancaster: MTP Press. 1976: 9()-3.

13 Tytgat GN, Huibregtse K. Schellekens PTA. Felt Kamp-Vroom TH. Clinical and immunological observations in a patient with late onset immunodeficiency. Gastroenterology 1979; 76: 1458-65.

14 Webster ADB, Kenwright S. Ballard J et al. Nodular lymphoid hyperplasia of the bowel in primary hypogammaglobulinaemia: study of in vivo and in vitro lymphocyte function. Gut 1977; 18: 364-72.

15 Wright SG, Tomkins AM. Quantitation of the lymphocyte infiltrate in jejunal epithelium in giardiasis. Clin Exp Immunol 1977; 29: 40)8-12.

16 Phillips AD. Small intestinal-mucosa in childhood in health and disease. Scand J Gastroenterol 1981: 16: $65-85$.

17 Owen RL, Nemanic PC, Stevens DP. Ultrastructural observations on giardiasis in a murine model. I. Intestinal distribution, attachment and relationship to the immune system of Giardia muris. Gastroenterology 1979; 76: 757-69.

18 Ridley MJ, Ridley DS. Serum antibodies and jejunal histology in giardiasis associated with malabsorption. $J$ Clin Pathol 1976; 29: 30-4.

19 Kahn LB, Noris BH. Nodular lymphoid hyperplasia of the small bowel associated with primary small bowel reticulum cell lymphoma. Cancer 1974; 33: 837-44. 
20) Shaw EB. Jr. Hennigar GR. Intestinal lymphoid polyposis. Am J Clin Pathol 1974: 61: 417-22.

21 Lamers CBBW. Wagener DJT. Assman KJM et al. Jejunal lymphoma in a patient with primary adult onset hypogammaglobulinaemia and nodular lymphoid hyperplasia of the small intestine. Dig Dis Sci 1980: 25: 553-7.

22 Seligmann M. Mihaesco E. Frangione B. Studies on alpha chain disease. Ann NY Acad Sci 1971; 190: 487-5()).
23 Bognel JC. Rambaud JC. Modigliani R et al. Étude clinique, anatamopathologique et immunochimique d'un nouveau cas de maladie des charnes alpha suivi pendant cinq ans. Rev Eur Etud Clin Biol 1972; 17: $362-74$.

24 Gams RA. Neal JA. Conrad FG. Hydantoin induced pseudo-pseudo-lymphoma. Ann Intern Med 1968; 69: $557-68$.

25 Whitehead R. Primary lymphadenopathy complicating idiopathic steatorrhoea. Gut 1968; 9: 569-75. 